\title{
Empirical Effective Bandwidth Estimation for IPTV Admission Control
}

\author{
Alan Davy, Dmitri Botvich, and Brendan Jennings \\ Telecommunications Software \& Systems Group, \\ Waterford Institute of Technology \\ Cork Rd., Waterford, Ireland \\ \{adavy, dbotvich, bjennings\} atssg.org
}

\begin{abstract}
We propose an admission control approach for IPTV in which decisions to admit new flows are based on effective bandwidth estimates calculated using empirical traffic measurement. We describe our approach to estimate the effective bandwidth required to satisfy given Quality-of-Service constraints on traffic and specify a simple admission control algorithm which uses this estimation to manage flow admissions. We present the results of a simulation study, employing real traffic traces for long-lived flows, which indicate that our algorithm ensures that an adequate, but not overly generous, amount of bandwidth is allocated to ensure that Quality-of-Service targets for accepted flows will be met. We also compare our algorithm with alternative approaches based on analytical estimation of effective bandwidth; we demonstrate that these approaches are weak at taking into account related Quality of Service constraints and thus reserve inappropriate amounts of bandwidth, leading to either increased QoS target violations, or underutilisation of resources.
\end{abstract}

Keywords: IPTV, admission control, effective bandwidth estimation, Quality of Service.

\section{Introduction}

Admission control is a technique used by service providers to ensure customers' traffic flows are allocated sufficient bandwidth to ensure service level agreements relating to packet-level Quality-of-Service (QoS) are maintained during periods of network congestion. The goal of the service provider is to guarantee QoS for accepted traffic flows while maximising bandwidth available for newly arriving flows. In the context of IPTV service delivery, admission control plays a vital role, as flows (streaming movies and TV programmes) are typically high-bandwidth and must meet stringent QoS targets, so bad admission decisions can significantly degrade QoS for all accepted flows.

A key characteristic of any admission control algorithm how well it predicts the level of resources a new flow requires so that its QoS targets can be met. If a new flow request is accepted, the outlined packet level QoS must be guaranteed on the 
new request, without affecting QoS of already admitted flows. Fundamental to this process is the prediction of required effective bandwidth (the minimum amount of bandwidth required by a traffic stream to maintain specified QoS related targets [1]) of the aggregated traffic following admission of the new flow. If the admission control algorthim predicts that aggregated effective bandwidth should the flow be accepted will be greater than the available bandwidth, the flow must be rejected. Clearly, the more accurate the estimation of effective bandwidth, the more effective the admission control algorithm operates as a whole. Current effective bandwidth estimation approaches are typically based on theoretical analyses of traffic properties (see for example $[1,2,3])$, based on simplyfying assumptions such as constant packet sizes and inter-arrival times.

In this paper we propose an admission control algorithm based on an empirical estimation of effective bandwidth, or empirical admission control (EAC). This technique enables the admission control algorithm to predict more accurately the required amount of bandwidth needed to ensure admitted aggregated traffic flows will maintain agreed packet-level QoS targets. The paper is organised as follows: §2 discusses background to effective bandwidth estimation and admission control techniques; §3 defines the admission control framework, outlining the effective bandwidth estimation and admission control algorithm. It also details four admission control algorithms for comparison: Parameter Based Admission Control (PBAC) [4]; Experience based Admission Control (EBAC) [5]; Measurement and Traffic Descriptor based Admission Control (MTAC) [6]; and the ideal admission control algorithm. §4 defines the IPTV architecture studied, discussing topology settings and limitations imposed on the service provider with regards to IPTV service delivery. In $\S 5$ we evaluate the performance of our algorithm to the aforementioned admission control algorithms and show that our empirical approach to estimation of effective bandwidth supplies the admission control algorithm with adequate information to make well informed decisions on whether to admit additional services to the admitted aggregate traffic or not. $\S 6$ draws conclusions and outlines areas for future work.

\section{Background and Related Work}

Effective bandwidth is defined as the minimum amount of bandwidth required by a traffic stream to maintain specified QoS related targets [1]. There are many methods of theoretically estimating this value for different traffic models, see for example $[1,2,3]$. These approaches depend on statistical representation of traffic sources, and use limited constraints on traffic properties such as constant packet size and inter arrival times. As this is an unrealistic representation of real traffic, we adopt an empirical approach based on packet trace analysis that is similar to those outlined in $[7,8]$. We believe empirical estimation of effective bandwidth can improve accuracy and thus improve admission control decisions over admission control algorithms dependent on theoretical estimation.

Admission control approaches can be broadly divided into two groups, namely: parameter based admission control, and measurement based admission control. Parameter based admission control (PBAC) [4] is based on the assumption that $a$ 
priori knowledge of the bandwidth requirements of each traffic source. This technique also requires exact knowledge of currently admitted requests and current available bandwidth. PBAC describes service requests via sets of traffic descriptors; a disadvantage of this approach is that a priori knowledge of the service request can not always be established (for example, often only the peak or mean rate of the service is known).

Measurement based admission control (MBAC) [9] is an approach where the admission control scheme makes decisions based on measurements taken in real time from the network. The approach attempts to learn the characteristics and requirements of flows admitted and bases future decisions on this knowledge. The advantage of this approach is that estimation of required effective bandwidth from aggregate flows can be predicted with greater accuracy, and no a priori knowledge of service requests is required. In general measurements such as mean throughput and variance of traffic aggregate are collected and used as input to analytic techniques for estimating effective bandwidth. The major disadvantage of this technique is that it is susceptible to measurement inaccuracies that lead to inaccurate traffic predictions.

Hybrid approaches have been developed to address the problems highlighted with PBAC and MBAC approaches. Examples include Experience Based Admission Control (EBAC) [5] and Measurement Based and a priori Traffic Descriptor Admission Control (MTAC) [6]. These hybrid techniques use both measurements taken from the network and knowledge of submitted traffic descriptors to predict future bandwidth requirements of services. The algorithm we propose can also be considered as a hybrid approach: we directly estimate effective bandwidth from available traces (without use of complex traffic models), and use these estimates in conjunction with peak rate values (a traffic descriptor) in the admission decision process. For evaluation purposes we evaluate our algorithm against the MTAC and EBAC algorithms.

\section{Admission Control Framework}

Our admission control framework uses an approach to estimating the effective bandwidth of traffic by analysis of collected packet traces. A set of directly measured effective bandwidth levels are taken for the admitted aggregate traffic over a number of time intervals. Based on this collection we predict the required effective bandwidth for the following interval. Admission of a new service request is based on the addition of the predicted effective bandwidth and the peak throughput of the service request taken from the supplied traffic descriptor.

\subsection{Empirical Estimation of Effective Bandwidth}

Effective bandwidth can be defined for different types of QoS targets; for example: delay targets, loss targets, or both delay and loss targets combined. Our effective bandwidth estimation process (introduced in [10]) can be applied to all types of QoS targets; however in this paper we address delay targets only. A delay target specifies both the nominal maximum delay experienced on the network and the proportion of 
traffic that is allowed exceed this maximum delay. A typical example of a QoS delay target is $(50 \mathrm{~ms}, 0.001)$ which means that only $0.1 \%$ of traffic is allowed to be delayed by more than $50 \mathrm{~ms}$. As effective bandwidth depends on the QoS target, for different QoS targets the effective bandwidth will vary.

Our effective bandwidth estimation algorithm is defined as follows. Let delay $y_{\max }$ be the nominal maximum delay and let $p_{\text {delay }}$ be the percentage of traffic which can exhibit delay greater than delay $\max _{\text {. }}$. We define effective bandwidth $R_{\text {eff }}$ of a traffic source for delay QoS target (delay max,$p_{\text {delay }}$ ) as the minimal rate $R$ such that if we simulate a FIFO queue with unlimited buffer and processing rate $R$, the percentage of traffic which will exhibit delay greater than delay $\max$ will be less than $p_{\text {delay }}$. To estimate the effective bandwidth of a particular traffic source on the network, we take a recorded packet trace of that source. We observe that if we simulate a FIFO queue (initially assumed to be empty) with the same inputted traffic trace for different queue rates $R_{1}>R_{2}$ and estimate the percentages $p_{1}$ and $p_{2}$ of traffic delayed more than delay $_{\max }$ for different rates respectively, then $p_{1} \leq p_{2}$. This means that the percentage of traffic, $p$, delayed more than delay $\max _{\max }$ is a monotonically decreasing function of processing rate $R$. Using this observation it is straight forward to design, for example, a simple bisection algorithm for a recorded packet trace to find the minimal value of a queue rate such that the percentage of traffic delayed more than delay ${ }_{\max }$ is less than $p_{\text {delay }}$. We use this process to estimate the effective bandwidth $R_{\text {eff }}$ of a collected trace, assuming all traffic within the trace is of the same traffic class, with a common QoS delay target. Note that we assume that all IPTV traffic for a given service provider will be subject to the same QoS targets and will therefore be assigned to the same traffic class.

\subsection{Admission Control Logic}

We assume that through provisioning and traffic engineering, $C_{\text {total }}$ bandwidth is available edge-to-edge for the traffic aggregate. When a new flow $f_{\text {new }}$ arrives it requests for a peak rate $R_{\text {fnew }}$. If (1) holds, admission is granted to $f_{\text {new }}$, otherwise the flow is rejected (2).

$$
\begin{aligned}
& \text { If }\left(R_{f_{\text {few }}}+R_{\text {eff predict }}\right) \leq C_{\text {total }} \text {, accept } \\
& \text { If }\left(R_{f_{\text {new }}}+R_{\text {eff predict }}\right)>C_{\text {total }}, \text { reject }
\end{aligned}
$$

The algorithm adds the requested flow peak rate to the predicted effective bandwidth of the already admitted traffic to establish if there is adequate bandwidth available for the service, while guaranteeing QoS targets on admitted traffic are maintained. The process of predicting the required effective bandwidth $R_{\text {eff rectic }}$ for the interval is as follows. Using the empirical effective bandwidth estimation algorithm and specified QoS target on packet delay, recorded packet traces are processed to find the adequate FIFO queue service rate that satisfies the QoS targets. The measured service rate or effective bandwidth $R_{\text {eff }}$ is recorded for a number of $N$ intervals within 
a set $\left\{R_{e f f}\right\}$, where $N=\#\left\{R_{e f f}\right\}$. From this set, the algorithm will choose the worst case effective bandwidth measurement to ensure a conservative prediction for the next interval.

$$
R_{\text {eff predict }}=\operatorname{MAX}\left\{R_{\text {eff }}\right\}
$$

The decision on taking the worst effective bandwidth estimate out of $\mathrm{N}$ estimated values is to ensure the algorithm operates conservatively. Alternative approaches could be employed to predict an appropriate effective bandwidth value, such as using a simple moving average, exponentially weighted moving average, or a time exponentially weighted moving average [11]. All these approaches can lead the algorithm to be less conservative, although the memory of the latter two approaches can be configured to lead to more conservative behaviour.

\subsection{Experience Based Admission Control Algorithm}

We compare the performance of our admission control algorithm to that of the Experience Based Admission Control (EBAC) [5]. EBAC operates by taking into consideration a measured peak to mean ratio of previous admitted traffic. Based on a collection of these measurements, the algorithm chooses an appropriate up to date reciprocal of this ratio $\varphi(t)$, known as the over provisioning factor, suitable for inclusion in its admission control logic. The peak measurements used within the algorithm are explicit peak rate traffic descriptors supplied by the service request.

To take QoS into consideration, the algorithm uses a limited approach of representing traffic as a maximum link utilisation threshold $\rho_{\max }$. This threshold is calculated using a well known queuing system method of estimating effective bandwidth [3] of traffic with constant packet size, and constant inter arrival times. Taking these considerations into account, the admission control algorithm is as follows. If

$$
r_{f_{\text {new }}}+\sum_{f \in F(t)} r_{f} \leq c_{l} \cdot \varphi(t) \cdot \rho_{\max }
$$

holds, admission is granted and $f_{\text {new }}$ joins $F(t) . r_{f}$ represents the peak rate of the newly arrived flow $f_{\text {new. }} F(t)$ are the set of all admitted flows at time t. $c_{l}$ is the limit capacity or the maximum provisioned bandwidth. The algorithm gains experience by using a complex approach to estimating the current over provisioning factor. Previous peak to mean ratios are calculated and stored in a time exponentially weighted moving histogram. By choosing the $95^{\text {th }}$ percentile of this histogram, the most up to date over provisioning factor can be calculated.

\subsection{Measurement and Traffic Descriptor-Based Admission Control}

We also compare the performance of our proposed algorithm to that of the MTAC algorithm [6]. MTAC estimates the required effective bandwidth of traffic based on the assumption that traffic arriving at the admission point follows Gaussian characteristics. Equation (5) defines a method of estimating the required effective bandwidth of the currently admitted traffic, where $m$ is the mean aggregate bit rate, $\sigma$ 
is the standard deviation of the aggregate bit rate and $\varepsilon$ is the upper bound on allowed queue overflow probability.

$$
C=m+a^{\prime} \sigma \text { with } a^{\prime}=\sqrt{-2 \ln (\varepsilon)-\ln (2 \pi)}
$$

The algorithm also specifies a precaution factor used to ensure the algorithm behaves more conservatively as the network approaches congestion; this factor is calculated as follows. A single reference flow is defined by a reference mean $m_{\text {ref }}$ and reference standard deviation $\sigma_{r e f}$. For the total link capacity $C_{\text {total }}$, the total number of reference flows $T_{r e f}$ that can be simultaneously admitted for a given target bound on $\varepsilon$ (the Packet Loss Ratio), is calculated. Using mean and standard deviation measurements collected, the total number of reference flows $N_{\text {ref }}$ within the admitted traffic is calculated. The precaution factor defines the relationship between estimated number of reference flows $N_{\text {ref }}$ within the measured traffic, and the total number of reference flows $T_{\text {ref }}$ allowed within the total link capacity $C_{\text {total }}$. In [8] the precaution factor also takes into consideration traffic with different QoS requirements than the reference flow; however, as all traffic within our scenario has common QoS requirements, we use a simplified precaution factor formula.

$$
P F=\left(N_{r e f} / T_{r e f}\right)
$$

Admission is simply based on whether the estimated bandwidth multiplied by the precaution factor is less than the total available bandwidth; if not, the flow is rejected.

$$
\begin{aligned}
& \text { If }\left(C_{\text {est }} \times P F\right) \leq C_{\text {total }}, \quad \text { admit } \\
& \text { If }\left(C_{\text {est }} \times P F\right)>C_{\text {total }}, \quad \text { reject }
\end{aligned}
$$

\subsection{Parameter Based Admission Control}

As a comparison to the worst case scenario, we model a PBAC [4] algorithm. The algorithms decision depends completely on the peak rate of services, supplied by the traffic descriptor. No measurement is involved in this process. On receiving a service request, the algorithm decides on admission if the following holds:

$$
r_{\text {new }}+\sum_{f \in F(t)} r_{f} \leq c_{l}
$$

$r_{f}$ is the peak rate of a requesting flow. $F(t)$ holds a set of all admitted flows. The algorithm tests whether the sum of all admitted flows plus the new requesting flow, is less than the level of reservable bandwidth $c_{l}$.

\subsection{Ideal Admission Control}

We additionally model an ideal admission control algorithm having complete access to network measurements and precise knowledge of traffic descriptors. On receiving a service request the ideal admission control is able to predict exactly the required resources of the aggregate if the service was admitted; using this knowledge it can decide whether admission is possible or not. 


\section{IPTV Service Delivery Scenario}

The scenario we use to evaluate our admission control algorithm is IPTV, which is defined as delivery of television related services over an IP network. We first discuss the relationships between business entities in the delivery of IPTV services. From the admission control perspective, the entities we are interested in are the customer, the service provider and the network operator. There have been a number of projects that have proposed business related scenarios $[12,13,14]$ for delivery of video-ondemand service over QoS enabled IP networks. Within these projects, the concept of wholesale bandwidth is used to describe the arrangement where the service provider leases QoS guaranteed bandwidth from the network operator, as governed by an agreed Service Level Agreement (SLA). In turn, the service provider delivers QoS guaranteed services to its subscribed customers, as governed by a service provider to customer Service Level Agreement (constrained by the limits of its own agreement with the network provider). Failure to meet these SLA targets usually results in loss of revenue through discounting. We define packet level QoS targets related to these agreements within our IPTV scenario and focus on how the service provider ensures its customers receive guaranteed QoS on traffic in line with outlined SLAs.

The service provider has a maximum amount of bandwidth available at its disposal. We assume that within this limit, traffic will be guaranteed QoS on packet delay as specified by the service provider to network operator SLA. For our scenario the service provider leases bandwidth from the network operator assuming that at peak times, $20 \%$ of its customer base will be using offered services, in particular unicast video-on-demand. As delivery of this service can consume a large quantity of bandwidth, if the assumed peak in service access is exceeded without adequate management of bandwidth access there will be degradation in QoS experienced by all customers, resulting in significant revenue loss. In times of increasing network load, routers employ techniques such as Random Early Detection (RED), or Weighted Random Early Detection (WRED), which, when throughput reaches a particular threshold within a traffic class, drop packets randomly to avoid congestion. Packets are dropped randomly within the traffic aggregate; therefore, since users are not distinguished between within the traffic aggregate, all users' flows will experience degraded QoS. This potential problem is particularly relevant for IPTV, given the relatively strict QoS targets - for example, a packet loss ratio of $1 \times 10^{-7}$ is specified by the DSL Forum [15]. The service provider therefore employs an admission control strategy to curb these losses, and maximise resource utilisation.

\subsection{Simulation Model}

Fig. 1 depicts a service provider, a network operator and a number of connected customers. Each customer is connected to the network through xDSL with a downstream maximum throughput of $24 \mathrm{Mbps}$. The service provider has a single point of connection to the network, through which all service traffic is aggregated. It is at this ingress point that the effective bandwidth of aggregated traffic is measured, and the service provider performs admission control on service requests. All traffic measurement is also performed at this point and we assume that the required network dimensioning and traffic engineering has been performed by the network operator to 


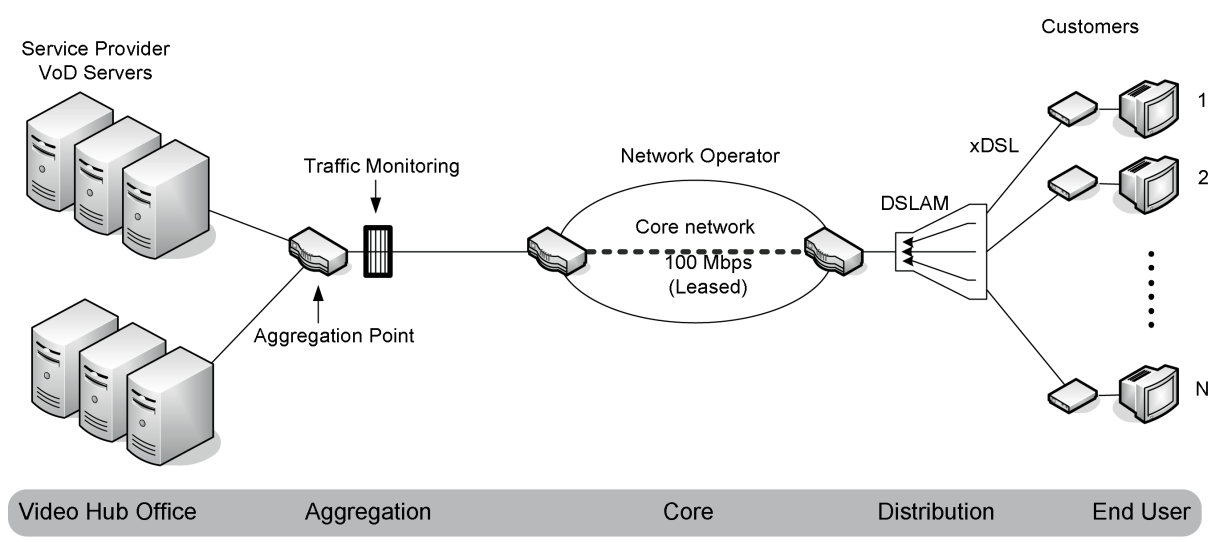

Fig. 1. IPTV Service Delivery Scenario

ensure that traffic within the service providers leased bandwidth capacity will be delivered within agreed SLAs. The service provider leases $100 \mathrm{Mbps}$ of bandwidth from the network operator. The service provider wishes to perform admission control on bandwidth up to $90 \%$ of this link to ensure there is a $10 \%$ margin of precautionary bandwidth available. To ensure all video traffic is treated with common QoS targets, they are aggregated into common traffic classes (we assume the DiffServ Code Point is used for this purpose).

We use a number of the video frame traces available at [16]. These traces are imported into OPNET ${ }^{\mathrm{TM}}$ and streamed across our simulated topology. These frame traces have been generated from several video sequences of typically 60 minutes length each. For the measurement of peak throughput per video, we use a precision of 0.05 seconds as this is approximately within the range of the QoS delay targets being imposed on the traffic. If larger precision were to be used, an inaccurate value may be calculated per flow, losing appropriate precision. For more information regarding the traces used, please refer to [17]. Table 1 shows the content (films) available for the customer to select from. A new service request (to view a film) is selected every 300 seconds, with each film having an equal probability of selection.

\section{Experimental Evaluation}

We argue that by using our approach for empirical estimation of effective bandwidth, our admission control algorithm does not require the use of complex over booking or precaution factors. The following experiments intend to evaluate our algorithm's performance with regard to QoS target violations and bandwidth utilisation in comparison to the PBAC, EBAC and MTAC and ideal admission control algorithms.

\subsection{Scenario Settings}

Films are chosen at random from the set of films offered by the service provider to its customers. Each film has a common Quality of Service target on packet delay of 
$(0.01 \mathrm{~s}, 0.0001)$ which outlines that only 0.0001 proportion of total traffic sent can be delayed by $10 \mathrm{~ms}$ or more. We look at the affect the admission control algorithms have on both traffic violations and bandwidth utilisation as service requests are submitted and accepted/rejected. For EBAC we need to set an appropriate maximum link utilisation threshold: in [3] the authors suggest setting a conservative and constant threshold value of 0.95 , which ensures that during congestion a maximum utilisation of $95 \%$ of the link is used. MTAC controls QoS through a Packet Loss Ratio (PLR) parameter; we specify a PLR of 0.0001, which matches our QoS target.

\subsection{Experimental Evaluation of Admission Control Algorithms}

Fig. 2 demonstrates the ratio between predicted required bandwidth and mean throughput for EAC in comparison to the MTAC, EBAC and PBAC algorithms. The relationship depicts how each algorithm reacts to the addition of multiple admitted flows. As flows are admitted, the empirical estimation diverges from a ratio of $\sim 12$ for one admitted, to 2 from multiple flows greater then 10. On the other hand, the EBAC estimation demonstrates a linear relationship as flows increase, as does the PBAC. This will results in under estimation of required bandwidth for smaller numbers of admitted flows, and in the case of PBAC, over estimation for higher numbers of flows. The MTAC algorithm actually increases demand requirements in proportion to measured mean, this is a result of the precaution factor that enables the algorithm to be more conservative as it approaches congestion.

Fig. 3 depicts the predicted effective bandwidth requirements for each admission control algorithm upon which it bases its admission decisions. Each vertical line within the graph represents a new service request arrival every 300 seconds. Table 2 shows the mean bandwidth utilisation and number of admitted flows, once AC algorithms reach their rejection threshold. We analyse the performance of each admission control algorithm through inspection of the results in Fig. 3 and Table 2:

- The Ideal admission control algorithm shows us that a maximum of 21 service requests can be admitted with a bandwidth utilisation of $47.99 \mathrm{Mbps}$, without experiencing QoS violations. It reaches its rejection threshold at 6000s.

- PBAC algorithm reaches its rejection threshold first, since the summation of peak service rates reach $90 \mathrm{Mbps}$ relatively quick (1800s). The algorithm results in a maximum bandwidth utilisation of $12.4 \mathrm{Mbps}$ and 6 admitted service requests in total. This has a relative error of $74 \%$ in comparison to the ideal. Whilst there are no QoS violations there is an unacceptable under utilisation of bandwidth.

Table 1. Characteristics of Video Traces

\begin{tabular}{|l|c|}
\hline Film & Peak Throughput (Mbps) \\
\hline Jurassic Park & 19.47 \\
\hline Mr Bean & 18.23 \\
\hline First Contact & 12.60 \\
\hline From Dusk till Dawn & 18.96 \\
\hline The Firm & 11.48 \\
\hline Die Hard III & 21.97 \\
\hline Aladdin & 21.27 \\
\hline
\end{tabular}




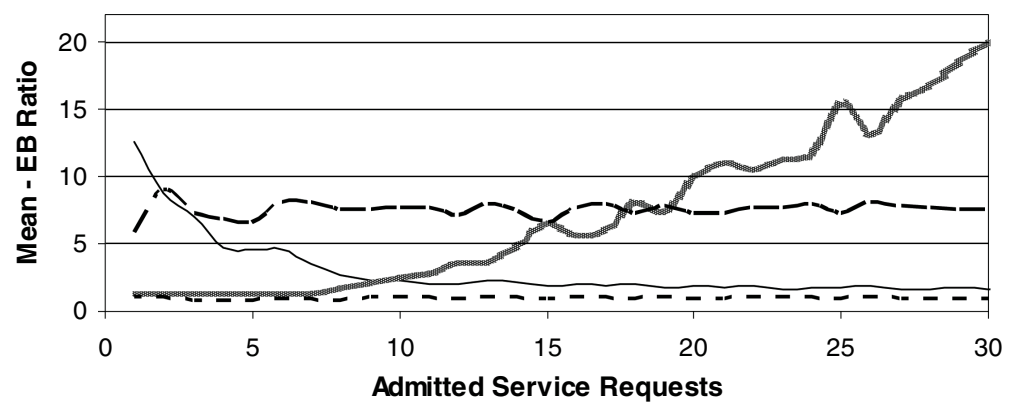

$\longrightarrow$ EAC MTAC - - - EBAC — - PBAC

Fig. 2. Mean / estimated effective bandwidth ratios for aggregate traffic

- MTAC reaches its rejection threshold next, since the precaution factor bares an impact on predicted bandwidth requirements at 3000s. Once the request at this point is accepted, the algorithm begins to become conservative allowing 12 service requests to be admitted. It too under utilises bandwidth at an average bandwidth utilisation of $22.67 \mathrm{Mbps}$ and error relative to the ideal of 52\%.

- EAC allows more services than MTAC to be admitted before reaching its rejection threshold at 4800s, whilst remaining free of any QoS violations on packet delay. This leads to a higher utilisation of bandwidth of $38.75 \mathrm{Mbps}$ and error relative to the ideal of $\sim 20 \%$, whilst still remaining relatively conservative with regards to QoS violations.

We note from Table 2 that the EBAC demonstrates the highest bandwidth utilisation of $77.33 \mathrm{Mbps}$, however as we see in Fig. 4 this is at a cost of incurring heavy QoS violations on traffic. EBAC, for our analysis scenario, is the only admission control algorithm that experiences QoS violations. Indeed, it performs the same as if no admission control algorithm was used. As the goal of the service

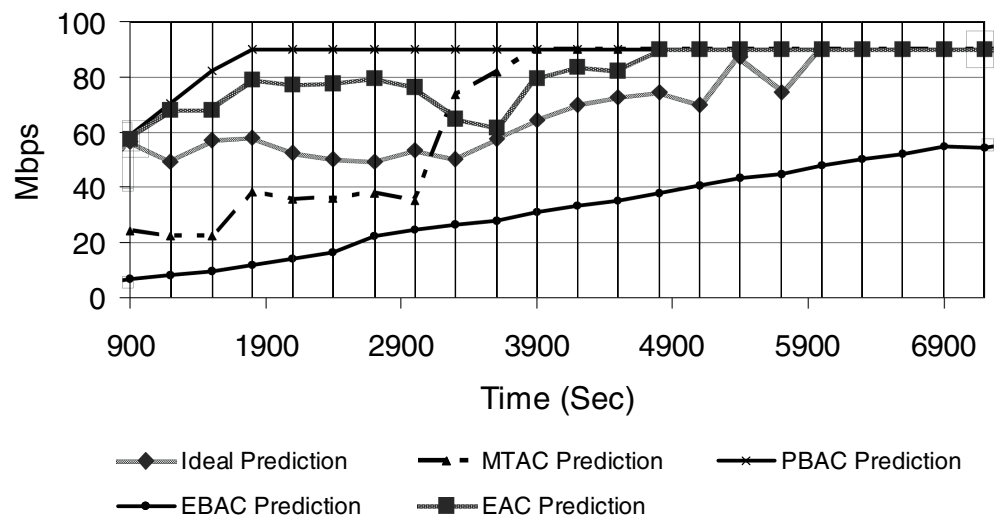

Fig. 3. Predicted required bandwidth for each admission control algorithm 
Table 2. Accepted Admissions for each Algorithm

\begin{tabular}{|l|c|c|}
\cline { 2 - 3 } \multicolumn{1}{c|}{} & $\begin{array}{c}\text { Accepted Service } \\
\text { Requests }\end{array}$ & Bandwidth Utilisation \\
\hline Ideal Admission & 21 & $47.99 \mathrm{Mbps}$ \\
\hline EAC Admission & 15 & $38.75 \mathrm{Mbps}$ \\
\hline MTAC Admission & 12 & $22.67 \mathrm{Mbps}$ \\
\hline PBAC Admission & 6 & $12.40 \mathrm{Mbps}$ \\
\hline EBAC Admission & 30 & $73.33 \mathrm{Mbps}$ \\
\hline No Admission Control & 30 & $73.33 \mathrm{Mbps}$ \\
\hline
\end{tabular}

provider is to maximise bandwidth utilisation while maintaining QoS targets, this algorithm falls considerably short in this scenario. QoS for this algorithm is controlled by the maximum bandwidth utilisation factor; the results here demonstrate that the value of 0.95 (as used for this simulation) is not sufficently conservative in relation to our QoS targets. If this algorithm were to take advantage of our proposed empirical approach to estimating effective bandwidth, we can see that the maximum link utilisation is $47.99 \mathrm{Mbps}$ of the $90 \mathrm{Mbps}$ (ideal AC), which in turn would set the threshold value to 0.53 , significantly less than the proposed value of 0.95 .

Based on these results we conclude that the EAC algorithm is most successful in addressing the service provider's goal of maximising bandwidth utilisation whilst minimising QoS violations. Table 2 indicates that our algorithm is quite close in performance to the ideal admission control solution: the difference in admissions averages approximately $20 \%$, which we believe is a reasonable degree of error. One significant advantage is that the algorithm is conservative in its predictions, so in times of congestion, the algorithm will not under provision services.

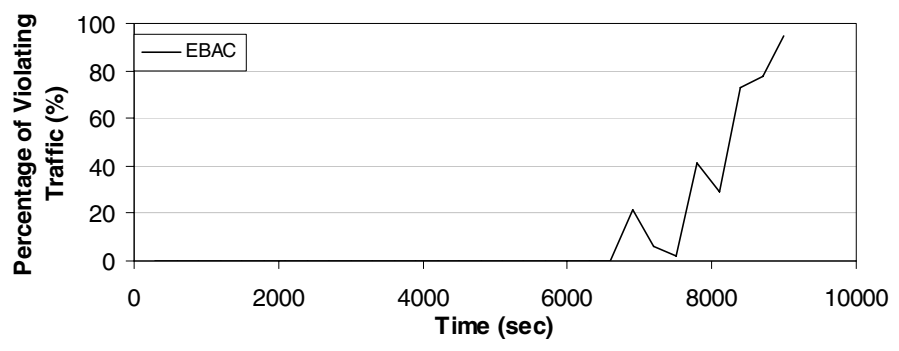

Fig. 4. QoS Violations after Admission Control for EBAC Algorithm

\section{Conclusions}

We have presented an admission control algorithm based on prediction of resources requirements using empirical estimation of effective bandwidth. We evaluated the algorithm through the use of an IPTV scenario with appropriate QoS targets on packet delay. IPTV was chosen as the basis of our study since the growing popularity of high bandwidth services like IPTV, which have strict QoS targets on packet loss and in turn delay, mean that effective QoS provisioning becomes ever more critical. 
Preliminary results demonstrate that our algorithm, EAC, is relatively accurate in predicting required effective bandwidth for admission control. It tends to be conservative in that it over estimates predicted effective bandwidth for the new aggregate in comparison to the ideal. We have also demonstrated our algorithm performs conservatively in comparison to the EBAC estimation algorithm, but EBAC incurs QoS violations due to underestimation of the required effective bandwidth on aggregate traffic. On the other hand, in comparison to a strict PBAC and MTAC, EAC utilises bandwidth more efficiently while remaining QoS violation free.

It is important that an admission control algorithm can react to varying degrees of QoS targets imposed on traffic. In future work we plan to evaluate the proposed algorithm with respect to varying QoS targets. We also wish to extent the algorithm with respect to IPTV services, by enhancing the decision process to incorporate IPTV related service information such as cost and duration of film content.

\section{Acknowledgements}

This work has received support from Science Foundation Ireland via the Autonomic Management of Communications Networks and Services programme (grant no. 04/IN3/I4040C) and the 2005 Research Frontiers project Accounting for Dynamically Composed Services (grant no. CMS006).

\section{References}

1. Kelly, F.: Notes on Effective Bandwidth, in Stochastic Networks: Theory and Application. In: Kelly, F.P., Zachary, S., Ziedins, I.B. (eds.) Royal Statistical Society Lecture Notes Series, vol. 4, pp. 141-168. Oxford University Press, Oxford (1996)

2. Norros, I., Roberts, J.W., Simonian, A., Virtamo, J.T.: The superposition of variable bit rate sources in an ATM multiplexer. IEEE Journal on Selected Areas in Communications 9(3), 378-387 (1991)

3. Roberts, J., Mocci, U., Vritamo, J.: Broadband Network Teletraffic - Final report of Action COST 242. In: Roberts, J., Virtamo, J.T., Mocci, U. (eds.) Broadband Network Traffic. LNCS, vol. 1155, Springer, Heidelberg (1996)

4. Fidler, M., Sander, V.: A Parameter Based Admission Control for Differentiated Services Networks. Computer Networks: The International Journal of Computer and Telecommunication Networking 44(4), 463-479 (2004)

5. Milbrandt, J., Menth, M., Junker, J.: Experience-Based Admission Control in the Presence of Traffic Changes. Academic Publisher, Journal of Communications 2(1), 1796-2021 (2007)

6. Georgoulas, S., Trimintzios, P., Pavlou, G.: Joint Measurement- and Traffic Descriptorbased Admisión Control at Real-Time Traffic Aggregation Points. In: ICC 2004. IEEE International Conference on Communications, vol. 4, pp. 1841-1845. IEEE Computer Society Press, Los Alamitos (2004)

7. Botvich, D.D., Duffield, N.: Large deviations, the shape of the loss curve, and economies of scale in large multiplexers. Queueing Systems 20, 293-320 (1995)

8. Baras, J.S., Liu, N.X.: Measurement and simulation based effective bandwidth estimation. IEEE Global Telecommunications Conference GLOBECOM 2004 4, 2108-2112 (2004) 
9. Lima, S.R., Carvalho, P., Freitas, V.: Distribution Admission Control for QoS and SLS Management. Journal of Network and Systems, Special Issue on Distributed Management 12(3), 397-426 (2004)

10. Davy, A., Botvich, D.D., Jennings, B.: On The Use of Accounting Data for QoS-Aware IP Network Planning. In: Mason, L., Drwiega, T. (eds.) ITC-20. LNCS, vol. 4516-0348, pp. 348-360. Springer, Heidelberg (2007)

11. Martin, R., Menth, M.: Improving the Timeless of Rate Measurements. In: PGTS. Proc of GI/ITG Conference on Measuring, Modelling and Evaluation of Computer and Communication Systems, pp. 145-154, ISBN 3-8007-2851-6, VDE Verlag (September 2004)

12. NetQoS, Policy Based Management of Heterogeneous Networks for Guaranteed QoS, Specific Targeted Research Project (STREP) from the 5th call of IST FP6 framework (June 18, 2007), last accessed www.netqos.eu

13. EuQoS, End-to-end Quality of Service support over heterogeneous networks, site last accessed (June 18, 2007), www.euqos.eu

14. MUSE, Multi Service Access Everywhere, IST 6th Framework Programme, site last accessed (June 18, 2007), www.ist-muse.org

15. DSL-Forum, Triple-play Services Quality of Experience (QoE) Requirements, Technical Report TR-126, Architecture \& Transport Working Group, Rahrer, T., Faindra, R., Wright, S. (eds.) (December 2006)

16. Telecommunications Networks Group, Technical University of Berlin, MPEG-4 and H.263 Video Traces for Network Performance Evaluation, site last accessed (June 18, 2007), www.tkn.tu-berlin.de/research/trace/trace.html

17. Fitzek, F.H.P., Reisslein, M.: MPEG-4 and H.263 Video Traces for Network Performance Evaluation (Extended Version). TU Berlin, Dept. of Engineering, Telecommunication Networks Group, Technical Report: TKN-00-06, (October 2000) 